\title{
Light Scattering and Current Enhancement for Microcrystalline Silicon Thin-Film Solar Cells on Aluminium-Induced Texture Glass Superstrates with Double Texture
}

\author{
Yunfeng Yin, ${ }^{1,2}$ Nasim Sahraei, ${ }^{1,2}$ Selvaraj Venkataraj, ${ }^{1}$ Sonya Calnan, ${ }^{3}$ Sven Ring, ${ }^{3}$ \\ Bernd Stannowski, ${ }^{3}$ Rutger Schlatmann, ${ }^{3}$ Armin G. Aberle, ${ }^{1,2}$ and Rolf Stangl ${ }^{1}$ \\ ${ }^{1}$ Solar Energy Research Institute of Singapore, National University of Singapore, Singapore 117574 \\ ${ }^{2}$ Department of Electrical and Computer Engineering, National University of Singapore, Singapore 117583 \\ ${ }^{3}$ Helmholtz-Zentrum Berlin für Materialien und Energie GmbH, PVcomB, Schwarzschildstrasse 3, 12489 Berlin, Germany \\ Correspondence should be addressed to Yunfeng Yin; yunfeng.yin@nus.edu.sg
}

Received 26 October 2014; Accepted 23 December 2014

Academic Editor: Francesco Bonaccorso

Copyright (C) 2015 Yunfeng Yin et al. This is an open access article distributed under the Creative Commons Attribution License, which permits unrestricted use, distribution, and reproduction in any medium, provided the original work is properly cited.

Microcrystalline silicon ( $\mu \mathrm{c}$-Si:H) thin-film solar cells are processed on glass superstrates having both micro- and nanoscale surface textures. The microscale texture is realised at the glass surface, using the aluminium-induced texturing (AIT) method, which is an industrially feasible process enabling a wide range of surface feature sizes (i.e., $700 \mathrm{~nm}-3 \mu \mathrm{m}$ ) of the textured glass. The nanoscale texture is made by conventional acid etching of the sputter-deposited transparent conductive oxide (TCO). The influence of the resulting "double texture" on the optical scattering is investigated by means of atomic force microscopy (AFM) (studying the surface topology), haze measurements (studying scattering into air), and short-circuit current enhancement measurements (studying scattering into silicon). A predicted enhanced optical scattering efficiency is experimentally proven by a short-circuit current enhancement $\Delta I_{\mathrm{sc}}$ of up to $1.6 \mathrm{~mA} / \mathrm{cm}^{2}$ (7.7\% relative increase) compared to solar cells fabricated on a standard superstrate, that is, planar glass covered with nanotextured TCO. Enhancing the autocorrelation length (or feature size) of the AIT superstrates might have the large potential to improve the $\mu \mathrm{c}-\mathrm{Si}: \mathrm{H}$ thin-film solar cell efficiency, by reducing the shunting probability of the device while maintaining a high optical scattering performance.

\section{Introduction}

Silicon thin-film solar cells are of industrial interest due to the fact that (1) the (hydrogenated) silicon layers are obtained directly from the gas phase (thus avoiding expensive crystallisation technologies and material consuming sawing technologies) and (2) the solar module production process can be directly integrated into the cell production process ("monolithic integration"). Hydrogenated microcrystalline silicon $(\mu \mathrm{c}-\mathrm{Si}: \mathrm{H})$, a mixed-phase material containing both amorphous and crystalline silicon regions, is widely used as a material forming the bottom cell of tandem or multijunction thin-film solar cells $[1,2]$. In order to reduce manufacturing cost and excess carrier recombination, the $\mu \mathrm{c}-\mathrm{Si}: \mathrm{H}$ absorber layer should be as thin as possible. However, due to the material's finite absorption coefficient, it is difficult to generate a sufficiently high photogeneration rate within the $\mu c-\mathrm{Si}: \mathrm{H}$ film for a single pass of the light through the film. Therefore, light scattering technologies have to be used to enhance the effective light path within the film [3-5]. With the help of light trapping methods, photogenerated currents of above $30 \mathrm{~mA} / \mathrm{cm}^{2}$ can be generated, using around $3 \mu \mathrm{m}$ thick $\mu \mathrm{c}-$ Si:H films, and very high PV efficiency has been reported [68]. The conventional thin-film light trapping is achieved by either etching the front transparent conductive oxide (TCO) layer of the solar cell prior to the $\mu \mathrm{c}$-Si:H deposition or growing the TCO layer with a self-organized surface texture [9]. This usually creates a nanotextured surface, with typical feature sizes ranging from several tens of nanometres to several hundred nanometres $[3,9,10]$. This surface texture generally provides good light trapping properties for wavelengths up to $650 \mathrm{~nm}$, but the ability to scatter near-infrared light from 700 
to $1100 \mathrm{~nm}$ is quite modest. To overcome this problem, surface textures with a larger feature size are needed [11]. Photolithography was used to create periodic honeycomb patterns with microscale size, and a significant improvement of nearinfrared light absorption was shown for substrate-type n-i-p $\mu c-S i: H$ thin-film solar cells $[7,12]$. For superstrate-type $\mathrm{p}-\mathrm{i}-\mathrm{n}$ $\mu \mathrm{c}-\mathrm{Si}: \mathrm{H}$ thin-film solar cells, texturing of the glass superstrate (leading to a microtextured glass surface) has been proposed recently, that is, using imprint-textured glass [11], rough glass by $3 \mathrm{D}$ texture transfer by ion etching [13], or aluminiuminduced texture (AIT) glass [14-16] in order to enhance light trapping. Besides, double-textured TCO, for example, using $\mathrm{HCl}$ and $\mathrm{HF}$ wet etching leading to microscale and nanoscale surface textures, respectively, was also proposed and demonstrated to improve the light trapping ability for the silicon thin-film solar cells $[17,18]$. In the present study, we use a double texture, that is, aluminium-induced texture glass superstrates (microtexture) covered with etched TCO (nanotexture), and compare its light trapping ability to a standard single-textured reference superstrate (i.e., planar glass covered with etched TCO). The AIT method enables us to obtain an industrially feasible microtextured glass surface with typical feature sizes in the $1-3 \mu \mathrm{m}$ range ("AIT glass"). It has already been theoretically proven, using numerical computer simulation, that a double-textured superstrate has an excellent light scattering potential for the entire wavelength range from 300 to $1100 \mathrm{~nm}$ because of the superimposed scattering behaviour [19]. Experimentally, a double-textured superstrate was investigated, using ion-etched textured glass in combination with a self-organised textured (MOCVD made) TCO growth, proving a short-circuit current enhancement $\Delta I_{\mathrm{sc}}$ of $1.5 \mathrm{~mA} / \mathrm{cm}^{2}$ compared to the planar glass/textured TCO reference superstrate [20]. Using AIT glass covered with etched TCO (double texture), we so far only investigated scattering into air (i.e., haze and angular resolved scattering), thereby highlighting the potential of a large $\Delta I_{\mathrm{sc}}$ enhancement using a double texture [16]. However, on a device level, this has not yet been proven. This demonstration is the scope of this paper.

In order to investigate the benefit of a double texture (i.e., the benefit of AIT glass when using TCO etching for light trapping), three different superstrates are used (see Figure 1): (1) planar glass covered with textured TCO ("single-textured" reference superstrate, REF), (2) AIT glass exhibiting a moderate correlation length $l$ covered with textured TCO, and (3) AIT glass exhibiting a large correlation length $l$ covered with TCO (i.e., two different "doubletextured" AIT glass superstrates, AIT-1 and AIT-2). The surface morphologies of these superstrates are studied by means of atomic force microscopy (AFM) and subsequent image processing. Thin-film $\mu \mathrm{c}-\mathrm{Si}: \mathrm{H}$ solar cells are processed on these textured superstrates and the resulting short-circuit currents $I_{\mathrm{sc}}$ are investigated using current-voltage $(I-V)$ as well as external quantum efficiency (EQE) measurements, in order to determine the optical scattering enhancement into silicon, if a double-textured superstrate is used. Furthermore, the influence of different AIT glass morphologies on the $\Delta I_{\mathrm{sc}}$ and on the solar cell efficiency, compared to the single-textured reference superstrate, is discussed.

\section{Experimental Details}

Three pieces of $10 \times 10 \mathrm{~cm}^{2}$ soda lime glass sheets $(3 \mathrm{~mm}$ thick) were used. Two of them were textured on one surface with the AIT method [14-16, 21], and the remaining one was not textured (reference). By changing the Al thickness, reaction time, and etching time, the surface morphology and the corresponding haze values of AIT glass sheets can be well controlled; see [16] for a detailed study. The surface morphology and visible light haze values were measured using an atomic force microscope (Veeco, model DI-3100 Nanoman) and a digital hazemeter (BYK haze guard, model AT-4725, light source: tungsten lamp). The spectrally resolved haze values (ranging from 400 to $1200 \mathrm{~nm}$ ) were measured by UV-VIS spectroscopy (Perkin-Elmer, Lambda 950). All three glass sheets (planar and textured) were cleaned with DI water and then coated with an aluminium-doped zinc oxide film ( $\mathrm{ZnO}$ :Al or "AZO"), using DC magnetron sputtering from a ceramic $\mathrm{ZnO}: \mathrm{Al}_{2} \mathrm{O}_{3}$ tube target. Next, the AZO films were etched in a highly diluted $\mathrm{HCl}$ solution, leading to a nanoscale surface texture on the AZO films. Thus, one single-textured reference superstrate (planar glass covered with nanotextured TCO) and two double-textured AIT superstrates (microtextured glass covered with nanotextured TCO) were processed [16]. All glass sheets were then cut into two parts. Half of them were used as superstrates for thinfilm solar cell fabrication, while half were used for surface morphology characterisation. For solar cell fabrication, the AZO coated glass sheets were cut into $5 \times 5 \mathrm{~cm}^{2}$ pieces. They were then attached to a $30 \times 30 \mathrm{~cm}^{2}$ stainless steel sample holder and loaded into a conventional RF $(13.56 \mathrm{MHz})$ PECVD system (Applied Materials, AKT 1600) for silicon thin-film deposition. Doped $\mu \mathrm{c}-\mathrm{SiO}_{x}: \mathrm{H}$ films, doped with either boron or phosphorus, were used as p-type or n-type hole/electron collecting layers of the $\mathrm{p}-\mathrm{i}-\mathrm{n}$ thin-film solar cells, with a target thickness in the range of $20-30 \mathrm{~nm}$ for the reference superstrate. The intrinsic $\mu \mathrm{c}$-Si:H absorber layer (target thickness of $1.75 \mu \mathrm{m}$ for the reference superstrate) was deposited at a temperature of $190^{\circ} \mathrm{C}$. It is emphasised that the resulting absorber layer thickness for the AIT glass superstrates is significantly lower $(\sim 1.5 \mu \mathrm{m})$, despite the fact that the same deposition run was used. This large thickness difference indicates a strong influence of the surface morphology on the thin-film deposition by PECVD, as, for example, highlighted in [22]. Next, a thin AZO film $(\sim 80 \mathrm{~nm})$ combined with a $150 \mathrm{~nm}$ thick silver layer was deposited onto the thinfilm silicon diode, serving as back surface reflector and rear contact of the solar cell. Finally, laser patterning was applied to define isolated cells with an area of $1.0 \times 1.0 \mathrm{~cm}^{2}$. The $I$ $V$ characteristics of the cells were measured under standard test conditions (AM1.5G spectrum, cell temperature $25^{\circ} \mathrm{C}$ ), using a class AAA dual-light-source solar simulator (Wacom, WXS_156S_L2). Cross-sectional images of the solar cells were taken by field emission transmission electron microscopy (XTEM, JEOL-JEM, 2010F) to determine their thickness. 


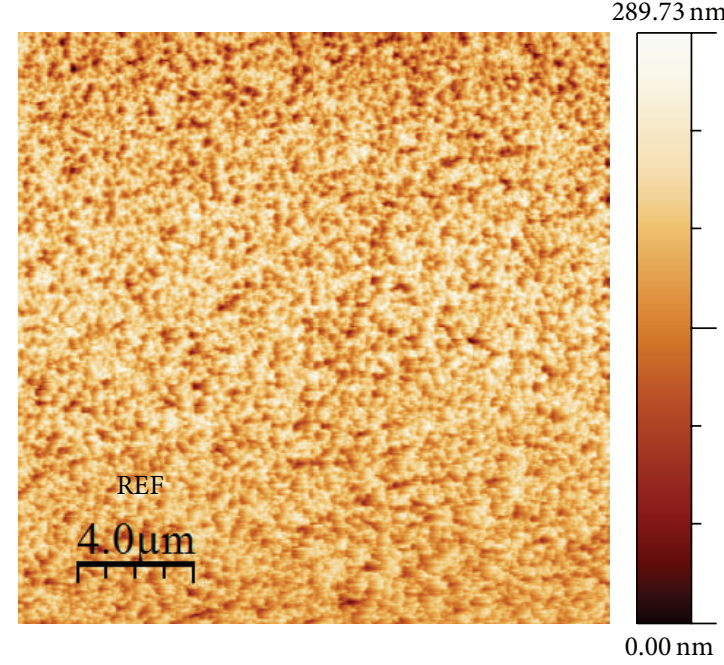

(a)

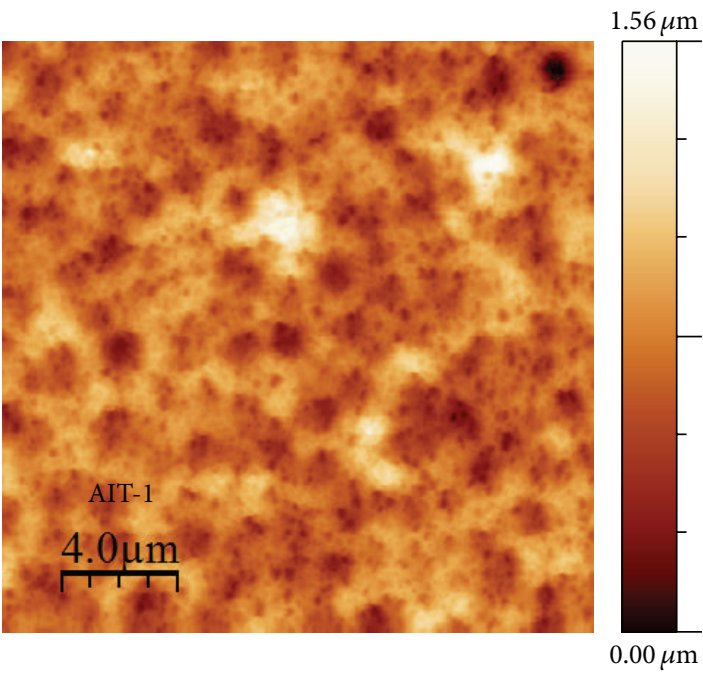

(b)

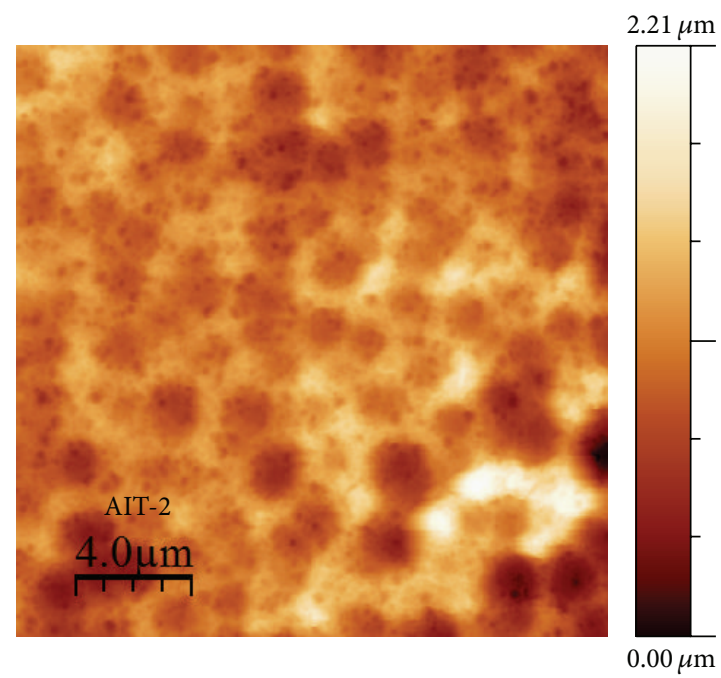

(c)

Figure 1: AFM images of the three different superstrates used in this study. (a) Planar glass superstrate covered with nanotextured TCO (reference superstrate); (b, c) microtextured AIT glass superstrate covered with nanotextured TCO. The AIT superstrate in (b) has an intermediate autocorrelation length $l$ (mean feature size), while that in (c) has a large autocorrelation length.

\section{Results and Discussion}

3.1. Surface Morphology and Haze of the Superstrates. The three different superstrates mentioned above were investigated by AFM. From the $20 \times 20 \mu \mathrm{m}^{2}$ AFM images as shown in Figure 1, surface morphology information such as mean surface roughness, surface angle distribution, and autocorrelation length were extracted (see Table 1) using the methods described in [23]. As compared to the reference superstrate (planar glass coated with nanotextured TCO), the AIT glass superstrates (microtextured glass coated with nanotextured TCO) show quite different characteristics. As expected, the AIT samples have much higher surface roughness (RMS) than the reference sample (about $200 \mathrm{~nm}$ versus $36 \mathrm{~nm}$ ). Also the mean feature size of the textured superstrates (i.e., the autocorrelation length $l$, which is a good indicator for the mean lateral feature size of randomly textured surfaces and which can be obtained from the autocorrelation function which can be extracted from AFM image data processing [23]; see Figure 2) differs considerably: for the reference superstrate the autocorrelation length is as small as $150 \mathrm{~nm}$, whereas the AIT-1 superstrate has a moderate autocorrelation length of about $750 \mathrm{~nm}$ and the AIT-2 superstrate has a large autocorrelation length of about $1050 \mathrm{~nm}$; see Table 1 . The autocorrelation function is an indicator of spatial persistency (or similarity) of the surface structure to itself at two positions of the surface. The distance between these two points is called lag length [24]. Assuming that the autocorrelation function can be presented by an exponential function, the autocorrelation length $l$ is defined as the lag length for which the autocorrelation function equals $1 / e(0.3678)$; see Figure 2 . As expected from the AFM images of the superstrates in Figure 1, 
TABLE 1: Measured haze values of visible light and calculated surface morphology parameters for the different superstrates used. With the exception of haze, all parameters were measured after TCO deposition and TCO texturing; that is, the AIT glass superstrates are doubletextured (microtextured glass covered with nanotextured TCO).

\begin{tabular}{lccc}
\hline & Reference & AIT-1 & AIT-2 \\
\hline Haze (no TCO) $(\%)$ & 0 & 50 & 70 \\
Haze (with TCO) $(\%)$ & 12 & 65 & 74 \\
RMS (nm) & 36 & 188 & 284 \\
Autocorrelation length $l(\mathrm{~nm})$ & 150 & 742 & 1050 \\
Average surface angle $\left(^{\circ}\right)$ & 20 & 40 (max at 30 and 50) & 40 (max at 30 and 50) \\
\hline
\end{tabular}

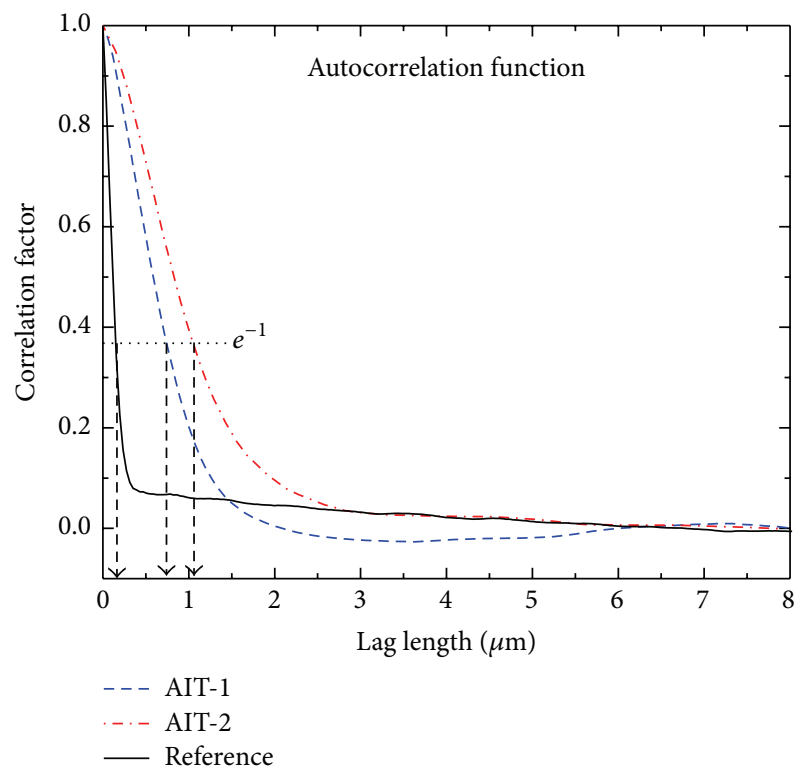

FIGURE 2: Determining the autocorrelation length $l$ for the three different superstrates used.

the autocorrelation length (the mean feature size) of the reference superstrate is much smaller than that of the AIT samples.

Surface angle distributions were extracted from the AFM images, as described in [23], specifying the probability of encountering a specific surface angle between 0 degrees (flat surface) and 90 degrees (surface perpendicular to the substrate plane); see Figure 3. Interestingly, AIT-1 and AIT2 have similar surface angle distributions; see Figure 3. Both superstrates exhibit an average surface angle of 40 degrees, with a pronounced maximum at 30 and 50 degrees, respectively. Based on the extracted surface morphology data, a simplified surface morphology model describing the three different superstrates is proposed; see Figure 4. Referring to this simplified surface morphology model, the reference superstrate is nanotextured with an autocorrelation length (mean self-repeating feature size) of $150 \mathrm{~nm}$ and an average surface angle about 20 degrees due to the texture. The AIT glass superstrates are double-textured (microtextured glass and nanotextured TCO). They have an autocorrelation length (mean self-repeating feature size) of $742 \mu \mathrm{m}$ or $1050 \mu \mathrm{m}$, respectively, an average surface angle of 40 degrees due to the microstructured glass, and an additional average surface angle of \pm 10 degrees due to the superimposed nanotexture

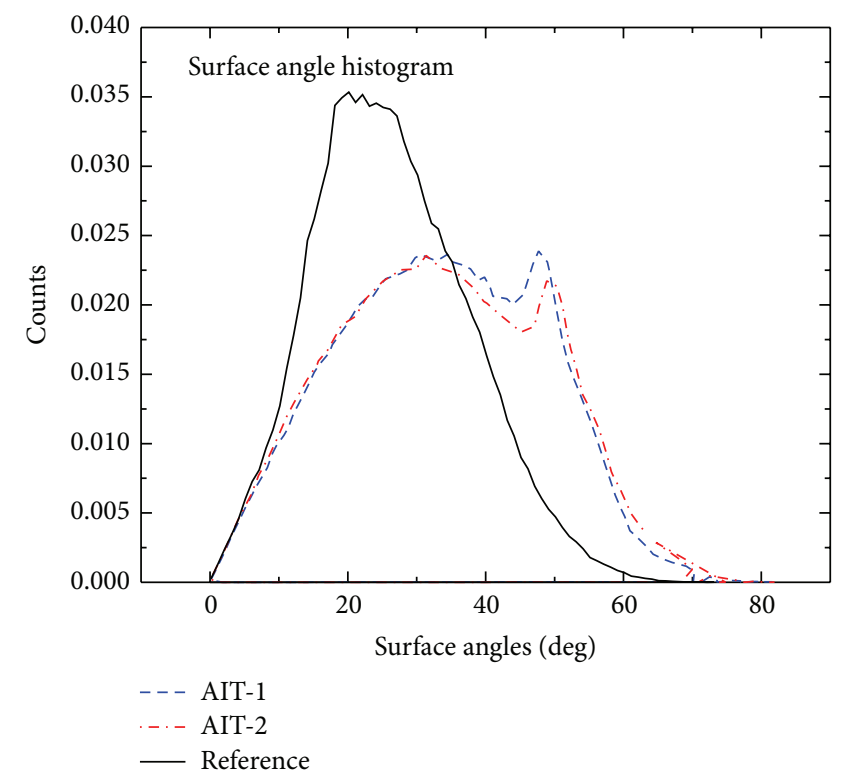

FIGURE 3: Surface angle distribution for the three different superstrates used.
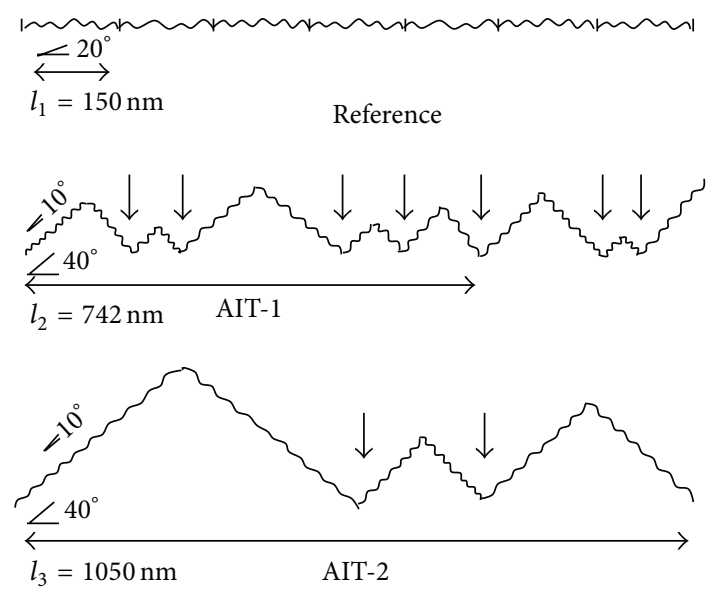

FIGURE 4: Schematic of the "simplified surface model" for the three different superstrates used.

stemming from the etched TCO. Thus, the average surface angle in the nanotextured surface valleys ("kinks") of the double-textured AIT glass superstrates is either 30 or 50 degrees; see Figure 4. Furthermore, the number of "kinks" as 
well as the surface roughness should correlate with the autocorrelation length $l$ of the AIT glass superstrates (i.e., fewer "kinks" and higher surface roughness for AIT superstrates with higher autocorrelation length $l$ ).

"Kinks" are often found to be the sources of microcracks and thus also sources for recombination active regions within microcrystalline silicon solar cells [25]. They can also be sources for local shunt formation [26]. The schematic model clearly reveals that, within a certain area, the AIT-1 superstrate (with moderate correlation length $l$ ) has a much higher density of "kinks" than the AIT-2 superstrate (with large correlation length $l$ ). This means that AIT glass superstrates with a larger autocorrelation length have a lower shunting probability for the fabricated $\mu \mathrm{c}$-Si:H thin-film solar cells. The AIT2 superstrate should therefore be less affected by local shunting, if used as a superstrate for $\mu \mathrm{c}-\mathrm{Si}: \mathrm{H}$ solar cell processing.

Haze measurements (diffuse scattering into air) for visible light $(400-700 \mathrm{~nm})$ were performed on the 3 superstrates, both before and after the application of the nanotextured TCO. Without TCO, the reference superstrate (planar bare glass) shows no haze at all, while the haze values of the two AIT superstrates vary significantly (50\% for AIT- 1 and $70 \%$ for AIT-2). After the application of nanotextured TCO, the haze values of the two AIT glass superstrates differ much less (65\% versus $74 \%$ ), despite the fact that the autocorrelation length of the two samples is significantly different (742 versus $1050 \mathrm{~nm})$. Compared to the haze of the reference superstrate $(12 \%)$, the haze of the AIT superstrates is much higher. Table 1 compiles the measured haze and surface morphology parameters of the three investigated superstrates.

Furthermore, the spectrally resolved haze was measured; see Figure 5. Considering $\mu \mathrm{c}-\mathrm{Si}: \mathrm{H}$ thin-film solar cells, the light scattering ability in the long-wavelength region (700$1100 \mathrm{~nm}$ ) is most important. As can be seen in Figure 5, the haze values of the double-textured AIT superstrates improve significantly in the long-wavelength region (compared to the single-textured reference superstrate). Therefore a stronger scattering of long-wavelength photons and thus a higher short-circuit current enhancement $\Delta I_{\mathrm{sc}}$ after $\mu \mathrm{c}-\mathrm{Si}: \mathrm{H}$ thinfilm solar cell processing can be expected for the AIT superstrates. Furthermore, it is expected that the AIT-2 superstrate will give a higher $\Delta I_{\mathrm{sc}}$ than the AIT-1 superstrate.

\subsection{Microcrystalline Silicon Thin-Film Solar Cells Grown on} the Different Superstrates Used. Several $1 \mathrm{~cm}^{2} \mu \mathrm{c}$-Si:H thinfilm solar cells were processed on the different superstrates discussed in the previous section. It is well known that the growth of $\mu \mathrm{c}-\mathrm{Si}: \mathrm{H}$ is surface morphology dependent $[26,27]$. As expected, the growth behaviour of $\mu \mathrm{c}-\mathrm{Si}: \mathrm{H}$ was considerably different when deposited on the double-textured AIT glass superstrates (microtextured glass covered with nanotextured TCO) compared to depositing on the single-textured reference superstrate (planar glass covered with nanotextured TCO), that is, the resulting film thickness differs significantly. The $\mu \mathrm{c}$-Si:H layers deposited onto AIT glass superstrates are thinner $(1.4-1.5 \mu \mathrm{m})$ compared to the layers deposited on the single-textured reference superstrate $(1.7 \mu \mathrm{m})$, keeping the deposition time constant (i.e., using the same deposition process); see Figure 6. This difference can be attributed to

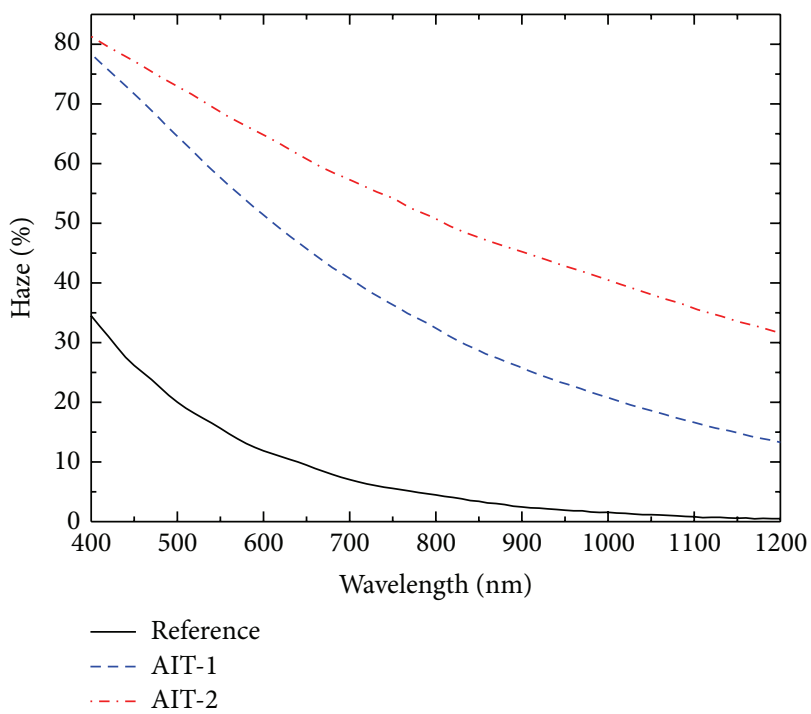

FIGURE 5: Spectrally resolved haze for the three investigated superstrates.

TABLE 2: One-sun $I-V$ parameters of the best $\mu \mathrm{c}-\mathrm{Si}: \mathrm{H}$ solar cells processed on the three investigated superstrates.

\begin{tabular}{lccccc}
\hline Superstrate & $J_{\text {sc }}\left(\mathrm{mA} / \mathrm{cm}^{2}\right)$ & $V_{\text {oc }}(\mathrm{mV})$ & $\mathrm{FF}(\%)$ & $\mathrm{Rsh}\left(\Omega \mathrm{cm}^{2}\right)$ & $\mathrm{Eff}(\%)$ \\
\hline REF & 20.1 & 487 & 64.9 & 440 & 6.4 \\
AIT-1 & 21.3 & 431 & 57.9 & 220 & 5.3 \\
AIT-2 & 21.7 & 475 & 59.9 & 270 & 6.2 \\
\hline
\end{tabular}

the fact that the AIT superstrates have a larger surface area (around 13\%) than the reference superstrate, stemming from the larger average surface angle of the microstructured AIT glass. Furthermore, the effects of shadowing and reemission should also be taken into consideration when depositing the films onto highly textured substrates [28].

Figure 7 and Table 2 show the corresponding one-sun $I-V$ curves and the extracted solar cell parameters of the best $\mu \mathrm{c}$-Si:H solar cells obtained on the three investigated superstrates. As expected, processing on double-textured AIT superstrates does improve the light scattering within the solar cell: a significant $\Delta I_{\mathrm{sc}}$ increase of 1.2 and $1.6 \mathrm{~mA} / \mathrm{cm}^{2}$ (5.8\% and $7.7 \%$ relative) was observed for the AIT- 1 and AIT-2 superstrates, respectively, compared to processing on the single-textured reference superstrate. Please note that the absolute short-circuit current density obtained for all solar cells is quite low $\left(\sim 21 \mathrm{~mA} \mathrm{~cm}^{-2}\right)$ compared to the current state of the art (up to $30 \mathrm{~mA} \mathrm{~cm}^{-2}$ ). This is due to three facts: (1) the processed $\mu \mathrm{c}-\mathrm{Si}: \mathrm{H}$ deposition used was optimized for micromorph tandem (a-Si:H/ $\mu \mathrm{c}-\mathrm{Si}: \mathrm{H})$ solar cell applications but not for single-junction $\mu \mathrm{c}-\mathrm{Si}: \mathrm{H}$ solar cells like those investigated in this study, (2) a comparatively thin $\mu \mathrm{c}-\mathrm{Si}: \mathrm{H}$ absorber layer thickness (1.4-1.5 $\mu \mathrm{m})$ was used, compared to a $3 \mu \mathrm{m}$ absorber thickness used in demonstrating an $I_{\mathrm{sc}}$ above $30 \mathrm{~mA} \mathrm{~cm}^{-2}[6,7]$, and (3) an industrial feasible $3 \mathrm{~mm}$ thick soda lime glass was used instead of, for example, a $1 \mathrm{~mm}$ thin Corning glass. As expected, the current enhancement is 


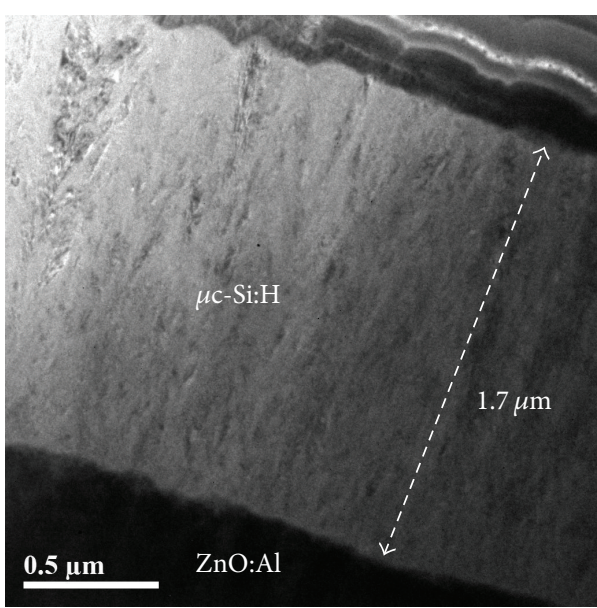

(a)

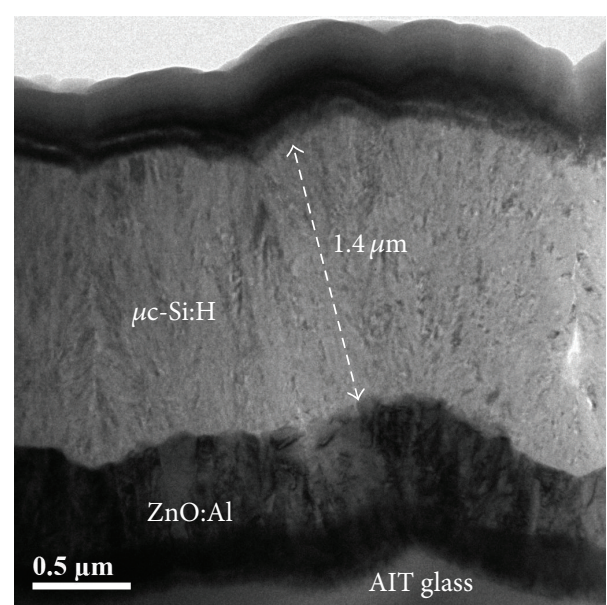

(b)

FIGURE 6: XTEM images of $\mu \mathrm{c}$-Si:H thin-film solar cells on (a) reference superstrate and (b) AIT glass superstrate.

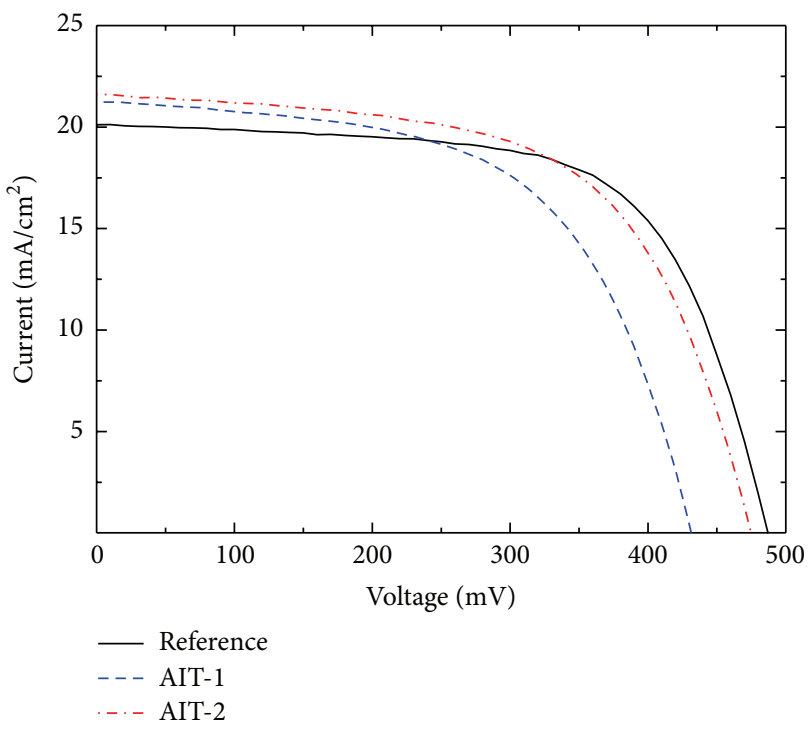

FIgURE 7: Measured one-sun $I-V$ curves of $\mu \mathrm{c}-\mathrm{Si}: \mathrm{H}$ solar cells processed on the three investigated superstrates.

mainly ascribed to the better light absorption for the infrared light (700-1100 nm) (see the EQE curves in Figure 8) since the haze values in the long-wavelength region improved significantly by using AIT glass superstrates, as shown in Figure 5. Furthermore, given the fact that the $\mu \mathrm{c}-\mathrm{Si}: \mathrm{H}$ film thickness on the AIT glass superstrates is thinner than on the reference superstrate, an even higher $\Delta I_{\text {sc }}$ increase can be expected if the film thickness is tuned to be identical. As expected, the AIT glass superstrate with the larger autocorrelation length (AIT-2) shows a higher $\Delta I_{\mathrm{sc}}$ increase (compared to AIT-1).

Thus, analysing only the measured $\Delta I_{\mathrm{sc}}$ using AIT glass superstrates clearly enhances the light scattering ability into silicon. However, analysing the resulting $I-V$ curves, so far, all cells processed (even including the cells processed on the reference superstrate REF) do suffer from significant shunting. As expected, this shunting issue is much more severe with

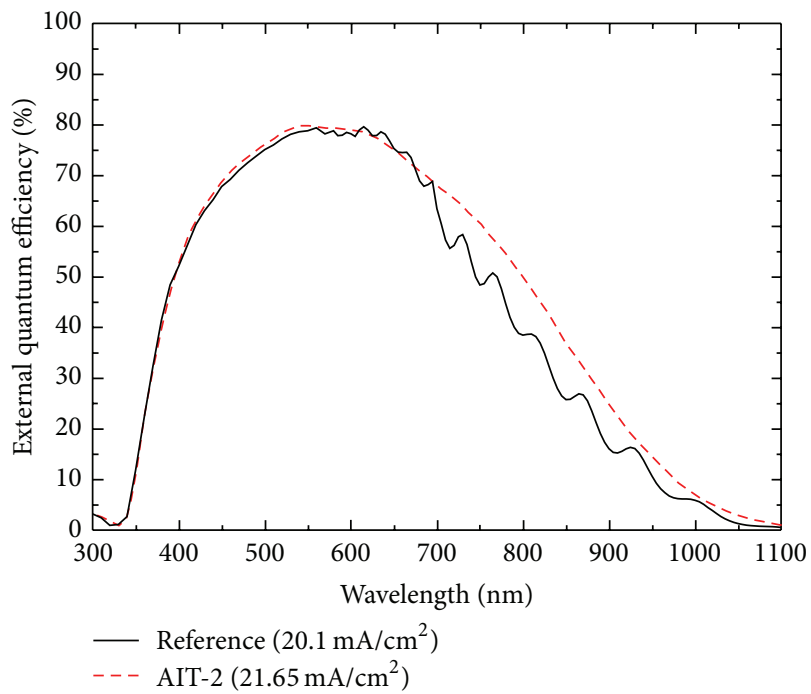

FIGURE 8: External quantum efficiency of $\mu \mathrm{c}-\mathrm{Si}: \mathrm{H}$ thin-film solar cells processed on the reference superstrate and on the AIT-2 superstrate (the corresponding short-circuit current is indicated in the legend).

processing on AIT glass superstrates. Thus a shunt-induced steep slope in the $I-V$ curves towards low voltages is observed (for all three superstrates), and in case of the AIT superstrates the low shunt resistance even affects the open-circuit voltage (and also the measured short-circuit current) of the solar cells; see Figure 7 and Table 2. In case of no shunting, the short-circuit current enhancement of the AIT superstrate $\Delta I_{\text {sc }}$ would be even higher than reported. Again, in agreement with our expectations outlined above, the AIT superstrate with the larger autocorrelation length $l$ (AIT-2) does suffer less from shunting compared to AIT-1. Indeed, defective areas ("cracks") were observed when depositing $\mu \mathrm{c}$-Si:H films on AIT glass superstrates, that is, above deep valleys ("kinks") which are induced by the AIT glass, as shown in Figure 9. 


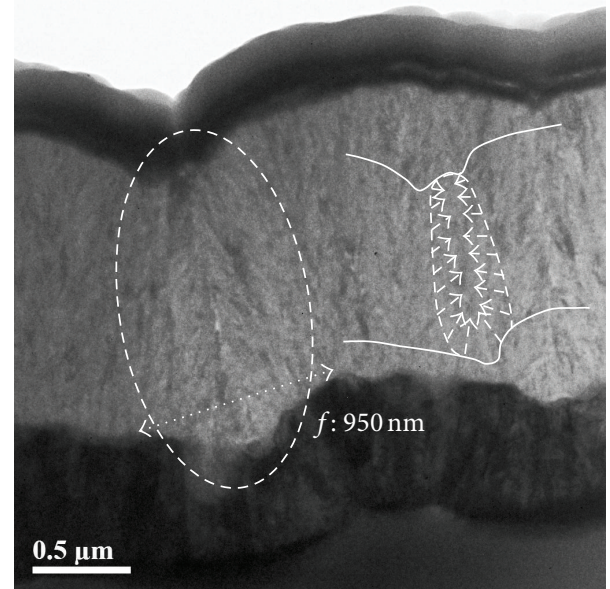

Figure 9: Measured crack or defective area formation (XTEM images) above deep valleys ("kinks") induced by the AIT glass.

Further optimisation still needs to be done, that is, (1) extending the autocorrelation length of AIT samples as much as possible, thus experimentally determining the maximum $\Delta I_{\text {sc }}$ due to the use of AIT superstrates and simultaneously reducing the shunting probability and (2) depositing different $\mu \mathrm{c}$-Si:H thin-films on the microtextured AIT superstrates, which are better adapted to grow shunt-free on a microstructured texture, that is, using lower-crystallinity films. Generally, $\mu \mathrm{c}-\mathrm{Si}: \mathrm{H}$ films with crystallinity of $50-60 \%$ are considered to be the best suited material for $\mu \mathrm{c}-\mathrm{Si}: \mathrm{H}$ solar cells [29], when deposited on conventional superstrates (i.e., suitable for our reference superstrate, planar glass covered with nanotextured TCO). However, when deposited onto highly textured substrates (i.e., on AIT glass), it is proposed to use lower-crystallinity films (40-50\%, with slightly poorer absorption of infrared wavelengths) or buffer layers [30]. Thus, it can be expected that by optimising the deposition conditions of $\mu \mathrm{c}$-Si:H films on AIT glass superstrates, as well as the AIT process itself, it should be possible to significantly reduce, or even eliminate, the local shunting problems experienced in our present experiments.

Based on the above analysis, $\mu \mathrm{c}-\mathrm{Si}: \mathrm{H}$ thin-film solar cells processed on AIT superstrates show a large potential to improve the solar cell efficiency, since (1) the improved light trapping (a higher short-circuit current density compared to a standard reference superstrate) has been experimentally proven and (2) a reduction/elimination of the presently observed local shunt formation seems possible.

\section{Conclusions}

Microcrystalline silicon ( $\mu \mathrm{c}-\mathrm{Si}: \mathrm{H})$ thin-film solar cells were processed on aluminium-induced texture (AIT) glass superstrates. The influence of the surface topology on the optical scattering behaviour and on the $\mu \mathrm{c}-\mathrm{Si}: \mathrm{H}$ film growth was investigated, by using a conventional single-textured reference superstrate (planar glass with nanotextured TCO) and two differently double-textured AIT glass superstrates (microtextured AIT glass covered with nanotextured TCO, exhibiting a moderate or large autocorrelation length, resp.). Surface topology information, such as surface roughness, surface angle distribution, and autocorrelation length (indicating the mean surface feature size), was extracted from AFM images. Scattering was investigated via haze measurements (scattering into air) and via the measured short-circuit current enhancement compared to the reference superstrate (scattering enhancement into the silicon). A significant haze increase was observed when using AIT superstrates, resulting in a short-circuit current enhancement of $1.6 \mathrm{~mA} / \mathrm{cm}^{2}$ (7.7\% relative increase). However, local shunt formation also emerged. A decreasing shunting probability was observed to correlate with an increasing autocorrelation length of the AIT superstrate. The experimental results predict that a further enhancement of the autocorrelation length (mean feature size) of the AIT superstrates shows a large potential for improving the solar cell efficiency, by reducing the shunting probability while maintaining (or even increasing) the light scattering abilities. Thus after optimization of the $\mu \mathrm{c}-\mathrm{Si}: \mathrm{H}$ thin-film deposition process on highly textured surfaces, the use of double-textured AIT glass superstrates has a large potential for improving the efficiency of $\mu \mathrm{c}-\mathrm{Si}: \mathrm{H}$ thin-film solar cells.

\section{Conflict of Interests}

The authors declare that there is no conflict of interests regarding the publication of this paper.

\section{Acknowledgments}

The Solar Energy Research Institute of Singapore (SERIS) is sponsored by the National University of Singapore (NUS) and Singapore's National Research Foundation (NRF) through Singapore Economic Development Board (EDB). This work was partly supported by the PVcomB (Helmholtz-Zentrum Berlin), Germany.

\section{References}

[1] A. Shah, J. Meier, E. Vallat-Sauvain et al., "Microcrystalline silicon and 'micromorph' tandem solar cells," Thin Solid Films, vol. 403-404, pp. 179-187, 2002.

[2] J. Yang, A. Banerjee, and S. Guha, "Triple-junction amorphous silicon alloy solar cell with $14.6 \%$ initial and $13.0 \%$ stable conversion efficiencies," Applied Physics Letters, vol. 70, no. 22, pp. 2975-2977, 1997.

[3] J. Müller, B. Rech, J. Springer, and M. Vanecek, "TCO and light trapping in silicon thin film solar cells," Solar Energy, vol. 77, no. 6, pp. 917-930, 2004.

[4] C. Haase and H. Stiebig, "Thin-film silicon solar cells with efficient periodic light trapping texture," Applied Physics Letters, vol. 91, no. 6, Article ID 061116, 2007.

[5] K. Söderström, F.-J. Haug, J. Escarré, C. Pahud, R. Biron, and C. Ballif, "Highly reflective nanotextured sputtered silver back reflector for flexible high-efficiency n-i-p thin-film silicon solar cells," Solar Energy Materials and Solar Cells, vol. 95, no. 12, pp. 3585-3591, 2011. 
[6] B. Yan, G. Yue, L. Sivec, J. Owens-Mawson, J. Yang, and S. Guha, "Correlation of texture of $\mathrm{Ag} / \mathrm{ZnO}$ back reflector and photocurrent in hydrogenated nanocrystalline silicon solar cells," Solar Energy Materials and Solar Cells, vol. 104, pp. 13-17, 2012.

[7] H. Sai, T. Koida, T. Matsui, I. Yoshida, K. Saito, and M. Kondo, "Microcrystalline silicon solar cells with $10.5 \%$ efficiency realized by improved photon absorption via periodic textures and highly transparent conductive oxide," Applied Physics Express, vol. 6, no. 10, Article ID 104101, 2013.

[8] S. Hänni, G. Bugnon, G. Parascandolo et al., "High-efficiency microcrystalline silicon single-junction solar cells," Progress in Photovoltaics: Research and Applications, vol. 21, no. 5, pp. 821826, 2013.

[9] J. Bailat, D. Dominé, R. Schlüchter et al., "High-efficiency PI-N microcrystalline and micromorph thin film silicon solar cells deposited on LPCVD ZNO coated glass substrates," in Proceedings of the IEEE 4th World Conference on Photovoltaic Energy Conversion (WCPEC '06), pp. 1533-1536, May 2006.

[10] M. Berginski, J. Hüpkes, M. Schulte et al., "The effect of front $\mathrm{ZnO}$ :Al surface texture and optical transparency on efficient light trapping in silicon thin-film solar cells," Journal of Applied Physics, vol. 101, no. 7, Article ID 074903, 2007.

[11] U. W. Paetzold, W. Zhang, M. Prömpers et al., "Thin-film silicon solar cell development on imprint-textured glass substrates," Materials Science and Engineering B: Solid-State Materials for Advanced Technology, vol. 178, no. 9, pp. 617-622, 2013.

[12] H. Sai, K. Saito, and M. Kondo, "Investigation of textured back reflectors with periodic honeycomb patterns in thin-film silicon solar cells for improved photovoltaic performance," IEEE Journal of Photovoltaics, vol. 3, no. 1, pp. 5-10, 2013.

[13] W. Zhang, E. Bunte, J. Worbs et al., "Rough glass by $3 \mathrm{~d}$ texture transfer for silicon thin film solar cells," Physica Status Solidi (c), vol. 7, no. 3-4, pp. 1120-1123, 2010.

[14] P. I. Widenborg and A. G. Aberle, "Polycrystalline silicon thinfilm solar cells on AIT-textured glass superstrates," Advances in OptoElectronics, vol. 2007, Article ID 24584, 7 pages, 2007.

[15] A. G. Aberle, P. I. Widenborg, and N. Chuangsuwanich, "Glass texturing," European Patent EP1613562 B1, 2011.

[16] S. Venkataraj, J. Wang, P. Vayalakkara, and A. G. Aberle, "Light scattering enhancement by double scattering technique for multijunction thin-film silicon solar cells," IEEE Journal of Photovoltaics, vol. 3, no. 2, pp. 605-612, 2013.

[17] M. Boccard, P. Cuony, C. Battaglia et al., "Nanometer- and micrometer-scale texturing for high-efficiency micromorph thin-film silicon solar cells," IEEE Journal of Photovoltaics, vol. 2, no. 2, pp. 83-87, 2012.

[18] M. Steltenpool, E. Moulin, F.-J. Haug et al., "Nano-imprint technology combined with rough TCO morphology as double textured light-trapping superstrate for thin film solar cells," in Proceedings of the 28th European Photovoltaic Solar Energy Conference and Exhibition (EU PVSEC '13), Paris, France, 2013.

[19] O. Isabella, J. Krč, and M. Zeman, "Modulated surface textures for enhanced light trapping in thin-film silicon solar cells," Applied Physics Letters, vol. 97, no. 10, Article ID 101106, 2010.

[20] A. Hongsingthong, T. Krajangsang, I. A. Yunaz, S. Miyajima, and $\mathrm{M}$. Konagai, " $\mathrm{ZnO}$ films with very high haze value for use as front transparent conductive oxide films in thin-film silicon solar cells," Applied Physics Express, vol. 3, no. 5, Article ID 051102, 2010.

[21] P. Vayalakkara, S. Venkataraj, J. Wang et al., "Aluminum induced glass texturing process on borosilicate and soda-lime glass superstrates for thin-film solar cells," in Proceedings of the 37th IEEE Photovoltaic Specialists Conference (PVSC '11), pp. 003080 003083, Seattle, Wash, USA, June 2011.

[22] M. Pelliccione, T. Karabacak, C. Gaire, G.-C. Wang, and T.-M. $\mathrm{Lu}$, "Mound formation in surface growth under shadowing," Physical Review B, vol. 74, no. 12, Article ID 125420, 2006.

[23] N. Sahraei, K. Forberich, S. Venkataraj, A. G. Aberle, and M. Peters, "Analytical solution for haze values of aluminiuminduced texture (AIT) glass superstrates for a-Si:H solar cells," Optics Express, vol. 22, no. 1, pp. A53-A67, 2014.

[24] J. M. Bennett and L. Mattsson, Introduction to Surface Roughness and Scattering, Optical Society of America, Washington, DC, USA, 1989.

[25] H. B. T. Li, R. H. Franken, J. K. Rath, and R. E. I. Schropp, "Structural defects caused by a rough substrate and their influence on the performance of hydrogenated nano-crystalline silicon n-i-p solar cells," Solar Energy Materials and Solar Cells, vol. 93, no. 3, pp. 338-349, 2009.

[26] M. Python, O. Madani, D. Dominé, F. Meillaud, E. VallatSauvain, and C. Ballif, "Influence of the substrate geometrical parameters on microcrystalline silicon growth for thin-film solar cells," Solar Energy Materials and Solar Cells, vol. 93, no. 10, pp. 1714-1720, 2009.

[27] Y. Nasuno, M. Kondo, and A. Matsuda, "Effects of substrate surface morphology on microcrystalline silicon solar cells," Japanese Journal of Applied Physics, Part 2: Letters, vol. 40, no. 4A, pp. L303-L305, 2001.

[28] T. Karabacak, "Thin-film growth dynamics with shadowing and re-emission effects," Journal of Nanophotonics, vol. 5, no. 1, Article ID 052501, 18 pages, 2011.

[29] Y. Mai, S. Klein, R. Carius et al., "Microcrystalline silicon solar cells deposited at high rates," Journal of Applied Physics, vol. 97, no. 11, Article ID 114913, 2005.

[30] T. Söderström, F.-J. Haug, V. Terrazzoni-Daudrix, X. Niquille, M. Python, and C. Ballif, "N/I buffer layer for substrate microcrystalline thin film silicon solar cell," Journal of Applied Physics, vol. 104, no. 10, Article ID 104505, 2008. 

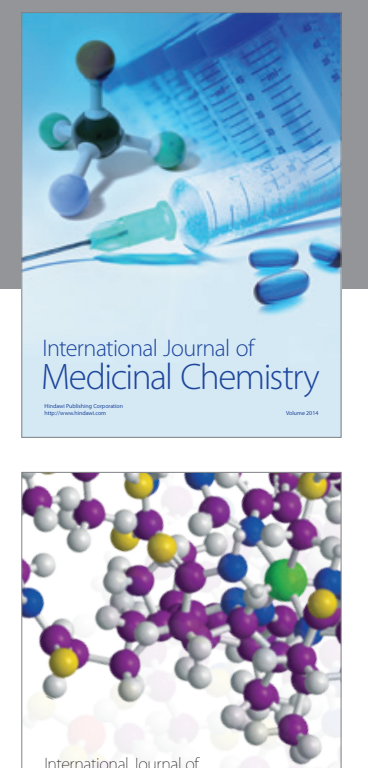

\section{Carbohydrate} Chemistry

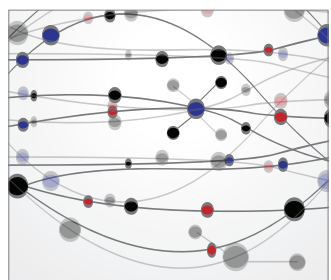

The Scientific World Journal
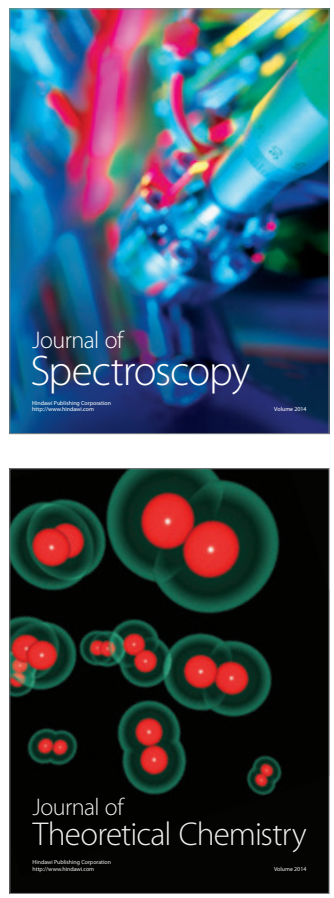
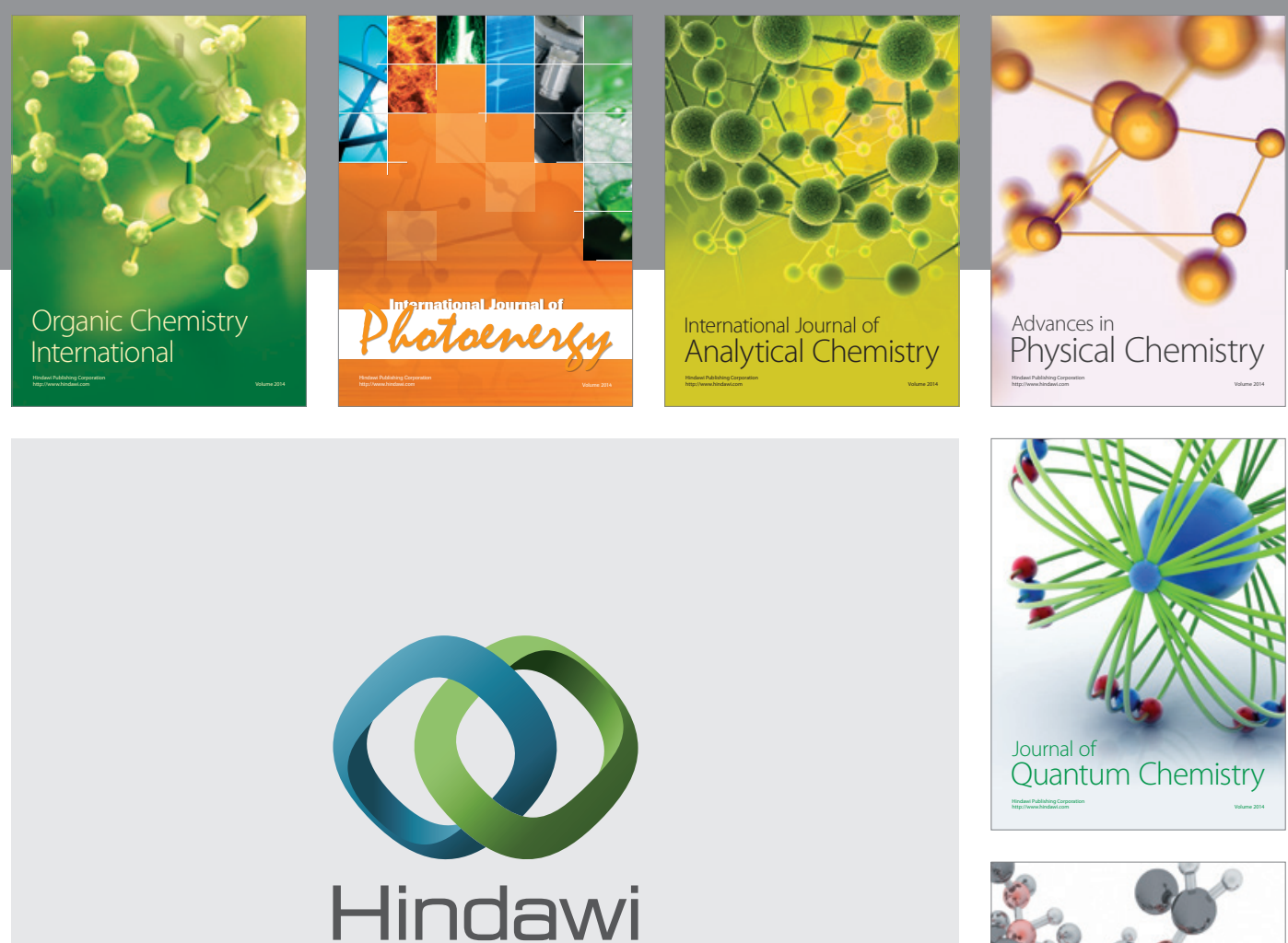

Submit your manuscripts at

http://www.hindawi.com

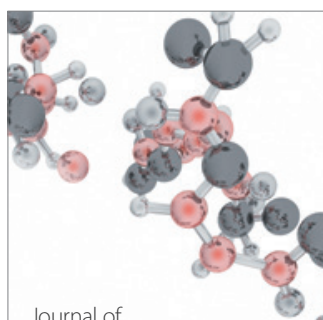

Analytical Methods

in Chemistry

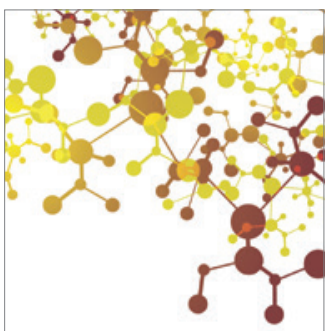

Journal of

Applied Chemistry

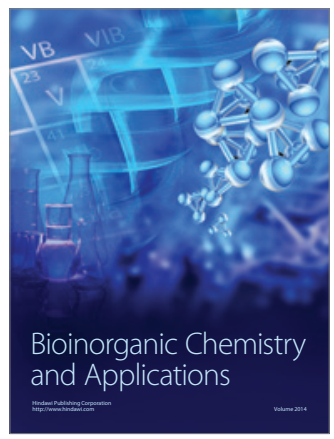

Inorganic Chemistry
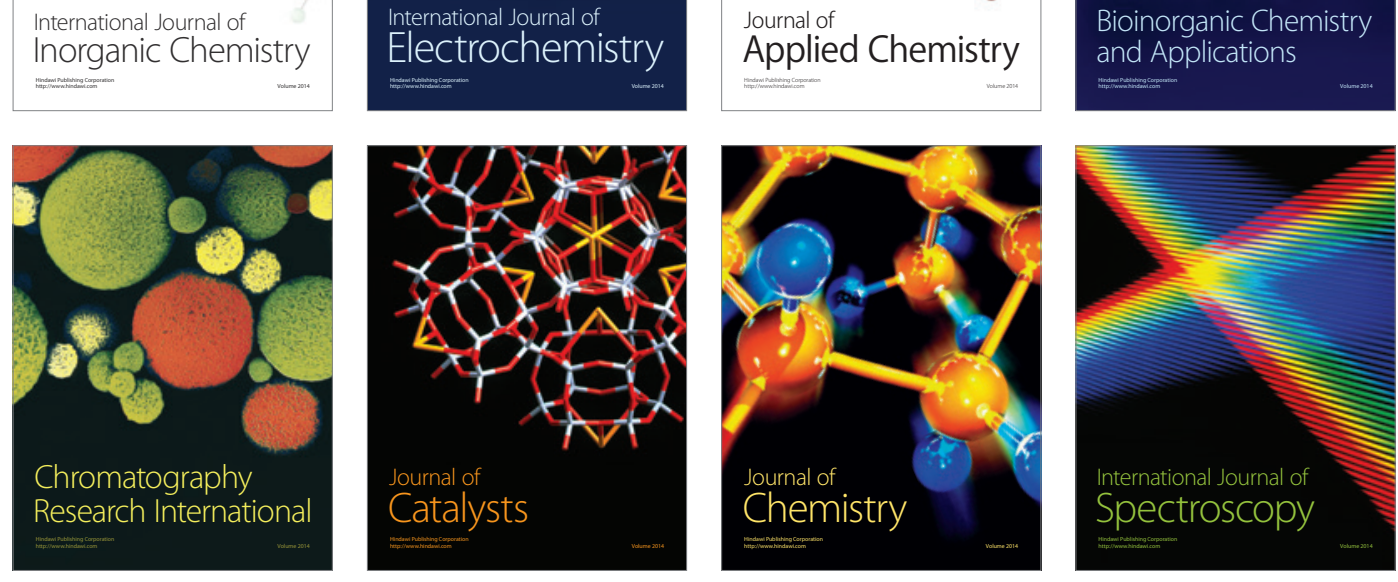